\title{
El papel dinamizador de las TIC en el aprendizaje extracurricular: formación específica e innovación educativa
}

\author{
Robert ARnAU Roselló \\ Universitat Jaume I de Castelló. Grupo de Investigación ITACA \\ rarnau@uji.es
}

\begin{abstract}
Resumen:
El impacto de las TIC convierte la docencia superior en una tarea prolongable más allá del espacio del aula. La visibilización de los resultados de la docencia es una labor en constante renovación para lograr hacer perceptibles las producciones audiovisuales, tanto para los alumnos como para el profesorado. Por todo ello, la oferta de un complemento formativo consistente en talleres y cursos específicos que motiven a los estudiantes a la hora de profundizar en algunas materias, es un elemento clave en la formación integral impartida en el área de conocimiento de Comunicación audiovisual, Periodismo o Publicidad.
\end{abstract}

Palabras clave: Formación específica; recursos digitales; extensión curricular; Trabajo colaborativo; Formación en TIC's.

\section{The dynamic role of ICT in extracurricular learning: specific training and educational innovation.}

\begin{abstract}
:
The impact of ICT teaching in higher education becomes an extendable assignment beyond the classroom space. The visibility of the results of teaching is a task that is constantly renewed to achieve perceptible audiovisual productions, both for students and teachers. Therefore, the offer of an educational supplement that consists of workshops and specific courses that encourage students to study some subjects in depth, is considered an essential element in the comprehensive training taught in the area of knowledge of Audiovisual Communication, Journalism or Advertising .
\end{abstract}

Key Words: Specific training; digital resources; educational extension; collaborative work; ICT in education.

\section{Referencia normalizada:}

Arnau Roselló, R. (2014): El papel dinamizador de las TIC en el aprendizaje extracurricular: formación específica e innovación educativa. Historia y Comunicación Social. Vol. 19. Núm. Especial Marzo. Págs. 27-36.

Sumario: 1. Introducción: la dinamización como principio, 2. Puesta en valor de la docencia a través de la visibilización y promoción de los resultados, 3. El LABCOM como motor de la formación específica: la oferta de cursos y talleres, 4 . Conclusiones 


\section{Introducción: La dinamización como principio}

La presente aproximación trata de acercarse a una descripción básica de diversas acciones, pese a su inicial invisibilidad, cuya puesta en marcha supone un elemento de primer orden en la construcción permanente del proceso de enseñanza aprendizaje. A partir del inicio de los estudios de grado en Comunicación Audiovisual en el cursos 2005-2006, con el recién inaugurado EEES, se propusieron en el seno del departamento de Ciencias de la Comunicación nuevos métodos para analizar las herramientas que la docencia práctica de las titulaciones proporcionaba a los alumnos y el impacto de dichas prácticas en su bagaje formativo.

El algunos casos, estas acciones, que permiten un acercamiento extracurricular de los estudiantes en aquellas materias que por su complejidad, transversalidad o especialización son potencialmente ampliables, suponen un reto de dinamización para el departamento, el laboratorio, e incluso, la propia universidad. El objetivo, pues, se situaba en aquellas especialidades en las que la TIC tuvieran un papel preponderante, en línea con las premisas de Lorente (Lorente, 1997: 56), debido al rol fundamental que desempeñan en la actual Sociedad de la Información

También, en sintonía con las indicaciones del Ministerio de Educación, se ha considerado fundamental, cumpliendo al mismo tiempo con algunas de las líneas maestras del naciente EEES, sustanciar determinados conocimientos esenciales de la formación en Comunicación Audiovisual trabajando sobre varios frentes simultáneamente.

Partiendo desde este punto, tal y como expresa acertadamente nuestro compañero Gómez Tarín (Gómez Tarín, 2009: 6), se ha procurado ser riguroso y aplicar una coordinación máxima entre cursos y materias para construir una línea de desarrollo continuo de los saberes y de sus aplicaciones en la práctica. De una parte, apuntalando aquellos contenidos prácticos que sirven al alumnado para obtener un conocimiento específico, muy útil en el sector laboral, con lo que se refuerzan sus posibilidades de acceso al exiguo mercado de trabajo existente hoy en día en nuestro país; de otra, procurando lograr la mayor visibilidad posible de los resultados de sus creaciones audiovisuales, a través de la participación de los alumnos y sus trabajos en eventos, festivales y plataformas colaborativas; por último, el refuerzo sistemático de la formación específica como un complemento indispensable de la oferta académica reglada, mediante la organización de cursos, talleres y seminarios especializados en las técnicas y las tecnologías audiovisuales emergentes.

En este contexto, las acciones a implementar se han concretado a partir de las tres líneas básicas de actuación que tradicionalmente se habían impulsado desde el departamento. En primer lugar, la línea de aprendizaje y prácticas en el seno de la titulación, es decir, aquella vía de especialización en la que se produce un uso de los saberes teóricos, extendidos de este modo a las prácticas de las asignaturas.

En segundo lugar, la línea de producción de audiovisuales fuera del horario lectivo, es decir, aquella a través de la cual se trata de estimular también la iniciativa del alumnado para generar sus propios discursos audiovisuales sin la intervención de 
las evaluaciones. Para ello, se diseñó un sistema de uso libre de los laboratorios para que cualquier proyecto, previamente supervisado por el profesorado y la dirección del LABCOM, pueda llevarse a cabo en régimen de producción asociada. En este caso, se exige al alumno una documentación exhaustiva que, en sí misma, supone una práctica adicional "de facto": Memoria del proyecto, Sinopsis, guión literario y guión técnico, Composición del equipo técnico y artístico, Plan de producción, Plan de rodaje, Presupuesto y esquema de comercialización, Registro de la propiedad intelectual, etc.

En tercer lugar, la línea de co-producción de audiovisuales con empresas del sector, es decir, aquella en la que la colaboración en proyectos de mayor envergadura con la participación del alumnado en producciones profesionales, es una realidad, siempre bajo la supervisión la coordinación del profesorado.

Cabe señalar que se ha trabajado desde una perspectiva cualitativa, más que cuantitativa. Se ha procedido analizando con mucho detalle las posibilidades efectivas de cada una de las acciones propuestas, para ofertar exclusivamente aquellas experiencias formativas que supusieran un estímulo real para alumnos y profesores, de modo que la motivación fuese un "extra" con el que contaran desde el principio los participantes del proyecto.

En este punto, es necesario hacer referencia a que la existencia de un Laboratorio dotado con infraestructuras audiovisuales de calidad (LABCOM) permite ofrecer complementos formativos, proyectando al mismo tiempo una imagen dinámica del departamento y abriendo una vía de difusión interesante que ofrece perspectivas de desarrollo especialmente sustanciales en algunas áreas de conocimiento como Comunicación Audiovisual, Publicidad o Periodismo.

\section{Puesta en valor de la docencia a través de la promoción y visibilización de los resultados: innovación y extensión del aprendizaje fuera del aula}

Antes de continuar con nuestra exposición, es urgente recordar el objeto de este proyecto. Los objetivos principales que animan la puesta en marcha de estas acciones se pueden sintetizar en: el estímulo de la creación de contenidos audiovisuales de los estudiantes de la Universitat Jaume I, la mejora en la calidad de dichos contenidos, el favorecimiento de un ambiente que estimule y motive a los estudiantes de las titulaciones de comunicación, la promoción de un contacto más estrecho con los profesionales de la producción audiovisual que participen en los cursos, y de las mejores producciones audiovisuales que se producen en la universidad, así como la difusión extrauniversitaria de las mismas.

De esta manera, las acciones que integran este proyecto se plantean en un momento crítico para las enseñanzas audiovisuales en toda la universidad española, en el que el posicionamiento de sus docentes va a ser fundamental de cara a sus aspiraciones 
futuras. Nos situamos precisamente ante la confluencia de dos fenómenos de gran trascendencia, que suponen una mutación de primer grado en el universo audiovisual, por una parte la expansión de productos audiovisuales de todo tipo, duración y formato a través de Internet, y por otra la globalización y el aumento del número de miembros del colectivo de profesionales de este sector. Por todo esto, en un momento de crisis económica e inquietud social como el que vivimos, se produce un doble movimiento: el desmoronamiento de lo que hasta el momento había sido un paradigma de la producción y la distribución audiovisual, y frente a ello, la emergencia de una gran creatividad en producciones independientes que se distribuyen por los nuevos canales contemporáneos, con la presencia inexorable de Internet como plataforma aglutinadora de contenidos audiovisuales o hipertextuales.

Desde la universidad, como institución al servicio de la sociedad y como foco de formación e investigación sobre la creación de contenidos audiovisuales, creemos que no debemos dejar pasar la posibilidad de apoyar a los estudiantes en la creación de lo que en la actualidad es uno de los principales pilares de nuestra cultura. El conocimiento encuentra los vehículos más eficaces para conducirse y potenciar las herramientas que la universidad posee para fomentarlo es una cuestión de coherencia, un deber.

A pesar de estas premisas, es evidente que la visibilización de los resultados prácticos de la docencia es una tarea compleja, que se mantiene actualizada a través de mecanismos diferentes, que abarcan diversos ámbitos de actuación y distintos procedimientos. La metodología empleada para tal propósito se basa en diferentes aspectos. Primero, un análisis bibliográfico que nos permita confeccionar el sustento teórico necesario para construir nuestra reflexión. En segundo lugar, el trabajo de campo, nos ayuda a iniciar líneas de trabajo eficientes. En un siguiente paso, en la ejecución administrativa del proyecto, en la organización y selección de las acciones a emprender.

A lo largo de estos últimos curso académicos (desde 2005) se han iniciado en el Departamento de Ciencias de la Comunicación un total de 10 proyectos de innovación en el área de conocimiento de Comunicación Audiovisual que han permitido cristalizar la labor de visibilización de los resultados y mejoras de la docencia cada curso. Además de los dirigidos por el autor de este texto desde el curso 2005 hasta el 2009 ("Diseño integral de un itinerario docente para el aprendizaje y el uso de los medios audiovisuales"), es necesario señalar el papel pionero y recopilador que ha desempeñado el proyecto dirigido por el vicedecano de la titulación de Comunicación Audiovisual, el profesor Gómez Tarín ("Coordinación integral de materias docentes con aplicaciones prácticas en producciones audiovisuales"), que se ha convertido en el referente de este tipo de acciones. Aún así, esta tarea es un compromiso múltiple, desde la perspectiva de que hay infinidad de elementos diversos que pueden contribuir a modificar la situación de invisibilidad y dar un nuevo impulso, hoy tan necesario, al proceso de enseñanza aprendizaje. 


\section{El labcom como motor de la formación específica: la oferta de cursos, talleres y seminarios}

Hay que destacar el papel seminal que desempeña el Laboratorio de Ciencias de la Comunicación (LABCOM) en todo el proyecto. El LABCOM es una infraestructura audiovisual de gran capacidad en la que se integran las tecnologías profesionales que tienen directamente relación son el sector audiovisual en su diversos ámbitos o variantes, como son: Fotografía (plató y laboratorio), Video (edición y rodaje), Televisión (emisión y realización multicámara), Audio (radio, doblaje y producción musical), y una amplia área multimedia que implica todas las especialidades anteriores. La existencia de esta infraestructura ha sido el origen de este proyecto, el puntal sobre el que descansa todo el planteamiento, sin ella no hubiese sido posible la organización de estas acciones formativas ni se hubiese configurado un equipo de trabajo como el que desarrolla sus actividades en la actualidad.

Una de las acciones fundamentales de esta estrategia de extensión de la docencia y complemento de la formación curricular reglada ha sido la puesta en marcha de una oferta de curso de formación específica que permitieran a los alumnos acceder a aquellas técnicas y tecnologías audiovisuales profesionales que por su carácter específico son objeto de tratamiento general en las asignaturas de las titulaciones. Como acción principal del proyecto, se encaminaba hacia la posibilidad de mantener una oferta de cursos que se renovara cada año

Hemos de tener presente que el LABCOM es una infraestructura que se usa como sustento técnico necesario para el desarrollo de las prácticas de las titulaciones impartidas en el seno del Departamento de Ciencias de la Comunicación. Sus actividades se desarrollan en las cuatro áreas tecnológicas principales implicadas en el sector profesional audiovisual, periodístico y publicitario: fotografía, video y televisión, radio y producción musical, y finalmente, redes y multimedia. De hecho, la estructura del préstamo en el LABCOM, se identifica absolutamente con los sistemas y procedimientos que se siguen en el ámbito profesional. La presencia de un almacén de prestamos y la responsabilidad en el uso y manejo del material audiovisual permite a los alumnos estar más familiarizados con los procesos propios del sector $\mathrm{y}$, de ese modo, relacionarse en primera persona con sistemas de funcionamiento que se asemejen al entorno profesional real.

La oferta de cursos es diseñada en función no sólo de las personas que participábamos en el proceso, sino también de los espacios y dispositivos con los que contamos en el LABCOM. Con estas condiciones de partida, el cometido de la oferta se condensaba, pues, en abarcar todo el espectro posible de tecnologías y tipos de curso, pero tratando de evitar caer en la dispersión y la atomización de una suma de ítems sin conexión entre ellos. Para ello, se plantea ofertar una tipología de cursos adaptada a las necesidades potenciales de nuestros usuarios, que pueda cubrir en tiempo y forma sus expectativas formativas. 
Se considera primordial ofrecer cuatro tipo básicos de cursos. Los cursos básicos, de 10 horas de duración media, plantean como una iniciación para inexpertos en las tecnologías audiovisuales, como un acercamiento a las cuestiones básicas de la técnica o especialidad en cuestión, como un elemento que permite quebrar la barrera que muchos usuarios creen tener en su relación cotidiana con las tecnologías. A través de estos CB se pretende acercar las bases de las tecnologías a aquellos estudiantes que necesiten una mayor dedicación técnica por falta de conocimientos o déficits en aspectos tecnológicos fundamentales.

De un nivel superior, los cursos específicos, esta vez con una duración de 10 a 20 horas de media, se dirigen a diversos aspectos particulares de ciertos desarrollos técnico-tecnológicos que pueden ser muy diversos. Se trata de aspectos muy concretos de cada ámbito tecnológico, en los que la especialización exige conocimientos absolutamente específicos relacionados con las propias disciplinas. Con estos CE se pretende aumentar el nivel técnico específico de los alumnos que ya poseen conocimientos tecnológicos muy centrados en determinadas materias.

En el siguiente grupo encontramos la oferta de cursos avanzados, de 20 o más horas de duración, que se concentran en las técnicas más evolucionadas, que requieren un nivel aún mayor de especialización por parte del alumno. Se acercan a aquellas tecnologías que por su reciente implantación, o su escasa expansión, son raramente accesibles en el entorno formativo. Por medio de estos CA se trata de dotar al alumnado de los conocimientos más avanzados que les permitan insertarse con ciertas garantías en el sector profesional correspondiente.

Para finalizar, los talleres, de unas 5 horas de duración media, se diseñan con una pretensión más cercana a la iniciación a las técnicas o disciplinas, pero desde un punto de vista histórico-estético. Consisten en una actividad introductoria a una temática de trabajo, con el mismo esquema metodológico para todos. Más que trabajar la técnica plantean una revisión histórica y estética y proponen una actividad práctica muy sencilla (desde un punto de vista práctico se componen de una exposición inicial de unos 90 min, una sesión práctica en exterior o en estudio de unas 3 horas, y una sesión de visualización análisis de los trabajos realizados por los alumnos de otros $90 \mathrm{~min}$.).

El diseño de otro tipo de cursos se ha pospuesto para una edición posterior de este proyecto, cuando se haya evaluado el impacto de la oferta de este año y acumulado datos suficientes como para realizar un análisis pormenorizado del alcance de la metodología empleada. De todos modos, dado que la voluntad del proyecto es continuista, las inserción de cursos de otro calado (más en relación con técnicas que con tecnologías) es cuestión de tiempo, en sucesivas ediciones se pretende incluir un abanico de cursos aún más amplio, con el fin de mejorar y completar la oferta progresivamente hasta abarcar todos los ámbitos tecnológicos posibles.

En suma, la oferta del LABCOM durante el curso 2012-1013, cuyo desglose se puede consultar en la página web http://www.uji.es/organs/vices/vpusl/labcap/, se ha construido en base a estas estructuras-modelos de curso como patrón a la hora de realizar una oferta tanto temática como metodológicamente equilibrada, teniendo en 
cuenta el peso específico de la fotografía y el video en sí mismos como tecnologías de uso mayoritario, y reflejando esta particular situación en una oferta compensada y adaptada a las necesidades formativas de los estudiantes de nuestras titulaciones (Comunicación Audiovisual, Periodismo, Diseño de Videojuegos y Publicidad y Relaciones Públicas).

El alcance del proyecto, la asistencia a los cursos y los resultados de la formación, en términos de evaluación de calidad de la docencia, quedan reflejados en un informe de estadísticas que genera el software de gestión del laboratorio, LABGEST. En él se recogen las evaluaciones de los alumnos asistentes a cursos y prácticas, los préstamos de material con sus respectivas incidencias y las reservas de espacios realizadas por los usuarios, con el fin de analizar, desde las diversas perspectivas que ofrecen las variables empleadas, la eficiencia y adecuación del sistema al entorno del departamento. De este modo, la mejora en la docencia y en la asistencia técnica de los cursos de formación así como de las actividades prácticas ejecutadas en el LABCOM, reposa sobre un análisis reflexivo de los datos extraídos de este proceso estadístico, lo cual resulta muy tranquilizador para el investigador-docente ya que las propuestas parten de unos trabajos analíticos rigurosos que garantizan sus capacidades aplicativas.

\section{Conclusiones}

Tras el repaso de estas acciones, cuyo objetivo principal se condensa en hacer de la formación específica un complemento sustancial de la docencia, es necesario, para acabar, proponer una evaluación del alcance y penetración de la propuesta. Esto nos dará una idea del "índice de calidad social" (Pérez Menayo, 2005: 63) que contiene esta iniciativa y de su grado de aplicabilidad en el entorno para el que ha sido específicamente diseñada.

Por ello, el desarrollo de las distintas propuestas se ha convertido en el motor principal de la innovación, ha permitido seleccionar aquellas que desde un punto de vista educativo son más atractivas para los estudiantes y al mismo tiempo ha servido de guía en las fases posteriores, cuando por distintos motivos se han recuperado estrategias, reflexiones, acciones o técnicas procedentes de propuestas que finalmente no habían sido puestas en marcha al principio. El proceso ha sido largo y con fases muy desiguales, pero durante su desarrollo todas las ideas han ido ocupando su lugar hasta encontrar acomodo en un proyecto ambicioso, pero realista, adaptado a las necesidades actuales del sector y a las perspectivas de los estudiantes. Cabe señalar no obstante que el periodo en el que se ha producido la convocatoria ha sido de un año completo, con dos convocatorias, una por semestre académico, lo que ha facilitado considerablemente la distribución de los cursos y el acceso a los mismo por parte del alumnado.

En general, la oferta ha tenido un impacto importantísimo en el Departamento de Ciencias de la Comunicación y el LABCOM. El total de asistentes a los cursos de 
formación impartidos ( 20 de los 25 ofertados) ha ascendido a 300 alumnos procedentes de las diferentes titulaciones del departamento de Ciencias de la comunicación (una media de 15 alumnos por curso), y el resultado de su evaluación ha sido de un promedio de 4'08 puntos sobre una escala máxima de 5, lo que ha supuesto una motivación añadida para el grupo del LABCOM impulsor de esta iniciativa y se ha entendido como un magnífico resultado que ofrece, no obstante, margen de mejora para convocatorias futuras. El éxito de la convocatoria no hay que buscarlo, sin embargo, en las acciones que se han implementado, sino en la motivación del grupo de estudiantes que ha seguido este itinerario formativo que se le proponía, sin ningún tipo de compensación en forma de evaluación, nota, puntos o ventajas sobre el resto de estudiantes, es decir, por puro interés y ganas de aprender (algo que el más avanzado sistema de enseñanza aprendizaje no puede sustituir).

La inercia que ha cogido el proyecto en esta primera edición, muestra la importancia del valor de la dinamización y constituye un impulso a todos aquellos proyectos que quieran desarrollar sus líneas de trabajo en direcciones similares a sumar sus propias mejoras y contribuir en el desarrollo permanente de la labor docente, sea en el área de conocimiento que sea. Y decimos en el área que sea, porque en prácticamente todas las titulaciones que se imparten en nuestra universidad se recurre a la producción de contenidos audiovisuales como una poderosa herramienta para complementar la docencia desde puntos de vista diferentes, aunque no excluyentes.

En el caso del LABCOM la tarea ha consistido en ir superando etapas que nos permitieran llegar a ofrecer un complemento formativo ajustado a las necesidades y posibilidades del servicio. El trabajo de los proyectos de innovación educativa se ha sumado a las tutorizaciones de prácticas independientes de las asignaturas de la formación reglada, al sustento técnico que implica el uso de una infraestructura audiovisual como el LABCOM, a la observación de unos métodos determinados de funcionamiento interno y finalmente, a la oferta de formación específica que culmina el itinerario previsto al inicio por los responsables de la iniciativa. En futuras convocatorias similares, cabe esperar que podamos reorganizar algunas de estas medidas mejoradas, una vez analizado su impacto en términos objetivables y estudiadas sus debilidades y fortalezas.

Para terminar, cabe señalar que la evaluación de la influencia del diseño e implementación de una oferta formativa completa y el alcance de las acciones que han permitido su ejecución, es muy positiva desde todos los puntos de vista. La motivación y el alto índice de matrícula en los cursos es un buen indicador de la salud y extensión de la convocatoria de este curso 2013. También el elevado índice de participación del profesorado y profesionales externos a la universidad garantiza un caudal de propuestas formativas para al menos 3 cursos más, dado la precaria situación del sector, es un buen momento para aprovechar el talento de profesionales que, por lo general, están participando en producciones y no tienen tiempo material para impartir estos talleres. Por lo que respecta a los resultados, hay que analizar de cerca dos factores de trascendencia incuestionable: la diversidad de estrategias es un elemento fundamental, por un lado, y la renovación de sus procesos es obligada, por otro. El 
estancamiento no permite cubrir las inquietudes renovadas de los estudiantes. Por tanto, la fluidez y eficiencia de las acciones escogidas para dinamizar la formación práctica ofrecida a los estudiantes está en relación directa con estas dos variables, siendo absolutamente efectivas en la generación del impacto necesario para promover puestas en cuestión del sistema empleado que desemboquen en mejoras y aumento de la adaptabilidad del modelo. Sólo si perseveramos y logramos conformar una oferta en constante evolución, podremos hacer de este proyecto una realidad permanente, que perdure en el tiempo y se convierta en un valor añadido al de las titulaciones que se imparten en la Universitat Jaume I.

\section{Bibliografía}

5.1 Libro, un autor

PÉREZ MENAYO, Vicente (2005): La calidad social, un nuevo paradigma en la Unión Europea. Madrid: Ministerio de Trabajo y Asuntos sociales

\subsection{Capítulo de libro}

ARNAU ROSELLÓ, Roberto (coord.) (2005): “Disseny integral d'un itinerari docent per a l'aprenentatge l'us de mitjans audiovisuals". En: Formació del professorat davant la convergència europea. V Jornada de millora educativa i IV Jornada de Harmonització. Castelló: Publicacions Universitat Jaume I.

GÓMEZ TARÍN, Francisco Javier (coord.) (2009): "Coordinación integral de materias docentes con aplicaciones prácticas en producciones audiovisuales". En: Metodologies centrades en l'estudiant a l'Espai Europeu d'Educació Superior. Actes de la VIII Jornada de millora educativa i VII d'harmonització europea. Castelló: Publicacions Universitat Jaume I.

LORENTE, Santiago (1997): "La sociedad de la información: su imagen, su proceso, sus logros e inconvenientes". Documentación Social, julio-septiembre.

\section{El autor}

Roberto Arnau Roselló es licenciado en Comunicación Audiovisual por la Universidad de Valencia y doctor en Comunicación por la Universidad Jaume I de Castellón. Ha impartido clases en el Instituto Universitario de Tecnología de la Universidad de Franche Comté (Besançon) donde ha llevado a cabo proyectos tecnológicos, docentes y de investigación. Su actividad se ha desarrollado tanto en el terreno de la docencia e investigación, con la publicación de artículos, capítulos de libro y participaciones en congresos, como en el del ejercicio profesional en el medio videográfico y radiofónico, en el que ha realizado múltiples obras audiovisuales y participado en numero- 
sas producciones asumiendo diversas funciones, como realizador, productor, director de fotografía, guionista, ayudante de dirección, etc. Sus líneas de investigación se centran en el análisis de la relación entre cine documental e intervención política, en las que se analiza el modo en que los planteamientos ideológicos y estéticos que hay detrás de cualquier representación interaccionan con los elementos expresivos de la imagen y su puesta en escena. Profesor Contratado Doctor del departamento de Ciencias de la Comunicación, es miembro del grupo de investigación ITACA UJI y Director del Laboratorio de Ciencias de la Comunicación de la Universitat Jaume I (LABCOM).

El presente estudio ha sido financiado con la ayuda del Proyecto de Investigación de la convocatoria Universitat Jaume I-Bancaja, con el título "Análisis de los flujos de transferencia de conocimiento entre los sistemas educativos superiores y la industria del videojuego", código 11I301.01/1, para el periodo 2012-14, bajo la dirección del Dr. Javier Marzal Felici 